\title{
Gestão Estratégica de Custos: Análise da Produção Científica na ABCustos no período de 2006 a 2015
}

Bacharelado em Ciências Contábeis pela Universidade do Oeste de Santa Catarina -
UNOESC
E-mail: rosane.pasa@hotmail.com

Sabrina Nascimento

Doutorado em Administração pela Universidade do Vale do Itajaí - UNIVALI Coordenadora do Curso de Ciências Contábeis da Universidade do Oeste de Santa

Catarina - UNOESC

Avenida Nereu Ramos, 3777-D. Seminário. Chapecó/SC. CEP: 89.813-000

E-mail: sabnascimento@gmail.com

Inocencia Boita Dalbosco Correio

Mestrado em Administração pela Universidade do Vale do Itajaí - UNIVALI Monitora do Programa de qualificação para exportação - PEIEX da Universidade do

Oeste de Santa Catarina - UNOESC

Rua Dom Carlos Eduardo Bandeira Saboia de Melo, 509-d. Bairro Presidente Médice.

Chapecó/SC

E-mail: inocencia.dalbosco@gmail.com.br

\section{RESUMO}

O estudo analisou as características da produção científica voltada à gestão de custos publicada na ABCustos, no período de 2006 a 2015, sob a perspectiva das redes sociais e da bibliometria. A pesquisa se caracterizou como descritiva e de cunho bibliográfico, com análise quantitativa dos dados. A amostra compreendeu 26 artigos selecionados por meio da ocorrência da terminologia "gestão de custos". Os resultados demonstraram que 24 artigos são de autoria múltipla e 61,5\% dos autores pertencem ao gênero masculino. A região sul concentrou $56 \%$ dos autores que publicaram na ABCustos. Os enfoques temáticos mais abordados foram custos, gestão, custos ambientais e contabilidade de custos. Quanto às redes sociais, o autor que ocupou a posição central da rede é o professor Jorge Eduardo Scarpin, com 4 artigos publicados. Entretanto, a rede social dos autores apresentou lacunas estruturais e laços que demonstraram a baixo compartilhamento de informações entre os 78 autores analisados. Verificou-se a predominância de pesquisas descritivas e qualitativas (54\%) e opção pelo método de estudo de caso (42\%). No que se refere à categoria referencial, os livros corresponderam a $53 \%$ das fontes de referências para construção da plataforma teórica dos estudos empíricos. Os achados evidenciaram necessidade aprofundar estudos que tratam da gestão de custos e apontaram um gap teórico na área contábil relacionada à necessidade de pesquisas empíricas voltadas à gestão de custos no contexto organizacional que subsidiem o processo decisório. 
Análise da Eficiência da Gestão de Custos do Processo de Produção de Arroz por pequenos e
médios produtores de Limoeiro do Norte - CE

Geanne Benevides Sá Negreiros, Rosângela Venâncio Nunes Rosângela Venâncio Nunes, Charles Washington Costa de Assis, Alexandra Alencar Siebra, Rita de Cássia Fonseca

Palavras-chave: Gestão de custos. Bibliometria. RedesSociais.

\section{Strategic Cost Management: Analysis of Scientific Production in ABCustos in the Period 2006 to 2015}

\section{ABSTRACT}

This study has aimed at analyzing the characteristics of scientific production about costs management published at ABCustos, from 2006 to 2015, through the perspective of social network and bibliometrics. The research is featured as descriptive and bibliographic, with quantitative analisys of data. The sample comprises 26 articles selected via the occurrence of terminology "costs management". The results show that 24 articles have multiple authors and $61,5 \%$ of the authors are of male gender. The South region has $56 \%$ of the authors who published at ABCustos. The most approached thematic focus were costs, management, environmental costs and costs accounting. As for social network, the author that features central position is Professor Jorge Eduardo Scarpin, with 4 articles published. However, the social network of the authors presents structural gaps and weak ties that show the lack of information share among the 78 authors analyzed. It was verified the predominance of descriptive and qualitative research (54\%) and option for the case study method (42\%). Regarding referential category, books correspond to $53 \%$ of the sources of references for the construction of theoretical platform of empirical studies. The findings evidenced the low depth of studies that approach costs management and pointed out a theoretical gap in the accounting field related to the need of empirical research towards costs management in the organizational context that subsidize the decision process.

Keywords: Costs management. Bibliometrics. Social network.

\section{INTRODUÇÃO}

A compreensão do custo de um determinado bem ou serviço tem o intuito de vislumbrar a continuidade e a sustentabilidade de qualquer entidade. A gestão dos custos contribui para a longevidade da organização, visto que possibilita uma análise sólida que subsidia o processo de tomada de decisão na organização, por meio das informações que disponibiliza para a administração da empresa. A contabilidade de custos pode ser utilizada não somente pelas indústrias, mas também pelas empresas 


\section{Análise da Eficiência da Gestão de Custos do Processo de Produção de Arroz por pequenos e médios produtores de Limoeiro do Norte - CE}

Geanne Benevides Sá Negreiros, Rosângela Venâncio Nunes Rosângela Venâncio Nunes, Charles Washington Costa de Assis, Alexandra Alencar Siebra, Rita de Cássia Fonseca

de prestação de serviço, comerciais e instituições financeiras, as quais passaram a experimentar seu potencial para o controle e a tomada de decisão (Schier, 2004).

Os sistemas utilizados pela contabilidade de custos são estruturados para mensurar e atribuir custos para os objetos de custeio. Entre as finalidades dessa contabilidade, destacam-se a avaliação dos estoques, o auxílio na determinação dos preços, o controle e a avaliação de desempenho de produtos, serviços ou unidades de negócio. Essas informações são utilizadas pelas organizações com vistas a subsidiar o processo de gerenciamento dos custos (Silva, 2010). Nessa perspectiva, a gestão estratégica de custos, conforme mencionam Ritta, Citadin e Pereira (2015), é um fator relevante para as organizações, pois faz uso das informações de custos no processo de gestão empresarial, o que possibilita a continuidade dos negócios.

Conhecer as pesquisas, ou seja, as produções científicas que abordaram essa temática podem auxiliar os gestores e pesquisadores a criarem um repositório de conhecimento que irá auxiliar no desenvolvimento de novas metodologias e soluções voltadas ao contexto organizacional.

Nesse sentido, com o intuito de conhecer as contribuições da gestão de custos no contexto organizacional, torna-se relevante realizar uma análise da produção científica que aborda o assunto, visto que a investigação desses estudos pode proporcionar novos insigths, geração de conhecimento e informações sobre o tema.

A análise da produção científica ou bibliométrica proporciona o entendimento no que diz respeito à gestão da informação e do conhecimento, pois é uma ferramenta estatística que permite mapear e gerar diferentes indicadores de tratamento. MaciasChapula (1998) entende que a bibliometria tem como objetivo estudar os aspectos quantitativos da produção, da disseminação e do uso da informação registrada.

Os estudos de natureza bibliométrica, conforme apontam Moraes Júnior, Araújo e Rezende (2013), geralmente são utilizados para quantificar dados referentes a um determinado assunto que está sendo discutido e também a uma determinada área. Esses têm a finalidade de mostrar as tendências da área estudada, demonstrar a forma como os estudos empíricos estão sendo realizados e o interesse dos pesquisadores no 
Análise da Eficiência da Gestão de Custos do Processo de Produção de Arroz por pequenos e médios produtores de Limoeiro do Norte - CE

Geanne Benevides Sá Negreiros, Rosângela Venâncio Nunes Rosângela Venâncio Nunes, Charles Washington Costa de Assis, Alexandra Alencar Siebra, Rita de Cássia Fonseca

assunto, bem como apontam os assuntos que se tornaram ultrapassados. Barboza, Souza, Nascimento, Bernardes e Castro (2016, p. 3) descrevem que "houve um aumento significativo das publicações relacionadas à bibliometria; este fenômeno ocorreu, principalmente, pela veiculação desses trabalhos por meio de periódicos e eventos científicos".

Alguns pesquisadores têm utilizado as redes sociais associadas a estudos bibliométricos. Para Tomaél, Alcará e Di Chiara (2005), as redes referem-se a agrupamentos; são fenômenos coletivos, ou seja, relacionamento de grupos, pessoas, organizações ou comunidades, denominados atores, e esses atores se classificam por laços fortes ou fracos.

Nessa perspectiva, investigar os estudos empíricos que abordam a gestão de custos se revela como um relevante instrumento para analisar o conhecimento resultante das pesquisas científicas que abordam o tema, sobretudo na Revista $A B C u s t o s$, já que este é um dos poucos periódicos especializados na área no contexto nacional, além de estar vinculado à Associação Brasileira de Custos ( $A B C)$. Esse periódico tem publicado estudos empíricos e teóricos com foco no desenvolvimento de conhecimento e aplicação de sistemas de gestão estratégica de custos que contribuem para a melhoria na qualidade de produtos, flexibilidade de processos, redução de lead time de custos, além de auxiliar as organizações que desejam tornar-se competitivas (ABCustos, 2016).

Desse modo, emerge a indagação que norteia o presente estudo: Quais as perspectivas das redes sociais e bibliométricas encontradas na produção científica voltada à gestão de custos publicada na Revista ABCustos, no período de 2006 a 2015? Para tanto, o estudo busca analisar a produção científica voltada à gestão de custos publicada na revista ABCustos, no período de 2006 a 2015, sob a perspectiva das redes sociais e da bibliometria. 
Análise da Eficiência da Gestão de Custos do Processo de Produção de Arroz por pequenos e médios produtores de Limoeiro do Norte - CE

Geanne Benevides Sá Negreiros, Rosângela Venâncio Nunes Rosângela Venâncio Nunes, Charles Washington Costa de Assis, Alexandra Alencar Siebra, Rita de Cássia Fonseca

\section{REVISÃO TEÓRICA}

Nesta seção, busca-se analisar os aspectos relacionados à base teórica que subsidia a pesquisa, como a gestão de custos, os estudos bibliométricos, as redes sociais e, ainda, os estudos anteriores que se assemelham à temática abordada.

\subsection{Gestão de Custos}

A contabilidade de custos tem sua origem a partir da contabilidade financeira, sendo que esta foi criada como instrumento para resolução de problemas de mensuração dos estoques e do resultado das organizações. No início, não era utilizada como ferramenta gerencial, porém, com o passar do tempo e com o crescimento das organizações, a contabilidade de custos passou a desempenhar um papel relevante no auxílio ao controle e à tomada de decisão (Schier, 2004).

No auxílio ao controle, os custos podem indicar determinados problemas ou situações não previstas que podem ocorrer por meio de comparações com padrões e orçamentos. Por outro lado, no auxílio à tomada de decisões, as informações de custos são relevantes para auxiliar nos processos decisórios das organizações (Bornia, 2010).

Os custos, conforme Martins e Rocha (2010), referem-se à ocorrência de consumo, à utilização ou à transformação de recursos econômicos de bens ou serviços no processo de produção, os quais envolvam outros bens ou serviços. Silva e Lins (2010, p. 7) comentam que "os custos são recursos consumidos no processo de produção de um bem ou serviço que se espera tragam benefícios atuais ou futuros para a entidade após a conclusão e venda do produto ou serviço".

Conforme Kohler (1952), apud Leone (2012), a contabilidade de custos é utilizada para o planejamento e execução de sistemas de custos, pois determina os custos de todos os segmentos utilizados para a realização de determinado produto ou serviço, envolvendo os departamentos, os centros de responsabilidades, entre outros. Além disso, estabelece padrões para determinação desses sistemas, manipula custos históricos, compara custos de diferentes períodos e custos reais com custos calculados, 

Análise da Eficiência da Gestão de Custos do Processo de Produção de Arroz por pequenos e
médios produtores de Limoeiro do Norte - CE

Geanne Benevides Sá Negreiros, Rosângela Venâncio Nunes Rosângela Venâncio Nunes, Charles Washington Costa de Assis, Alexandra Alencar Siebra, Rita de Cássia Fonseca

determina custos de alternativas, interpreta e apresenta informações de custos como auxilio à gerencia no controle e tomada de decisões das operações correntes e futuras.

Por fim, a contabilidade de custos tem por objetivo a coleta e fornecimento de informações que auxiliem na tomada de decisão de todos os tipos, seja relacionada com operações repetitivas ou de natureza estratégica, ajudando na formulação das principais políticas das organizações (Leone, 2012).

Dessa forma, as empresas necessitam de um sistema de gestão de custos que consiga mensurar e alocar os custos aos produtos da forma mais exata possível, para calcular o custo unitário total de cada produto, assumir o custo unitário total como resultante da soma dos custos variáveis aos custos fixos, ou a soma dos custos diretos aos custos indiretos atribuídos aos produtos (Wernke, 2005).

Nesse sentido, foram criados sistemas de custeio para prestar informações mais consistentes para o processo de tomada de decisão, ou seja, fornecer instrumentos a fim de que os usuários do sistema de custos pudessem obter informações confiáveis, relevantes e oportunas (Silva \& Lins, 2010).

$\mathrm{Na}$ literatura, são encontrados alguns métodos de apropriação de custos, como o sistema de custeio variável ou direto, sendo apropriados aos produtos e serviços somente os gastos a eles associados, os quais são classificados como custos diretos ou custos variáveis e despesas variáveis. Os demais gastos -classificados como custos indiretos, custos fixos e despesas fixas - não devem ser considerados no custeamento dos produtos e serviços (Wernke, 2005).

Em relação ao custeio por absorção, esse método consiste na apropriação de todos os custos de produção aos produtos elaborados por meio dos rateios (Santos, 2011). Destaca-se que o método é derivado da aplicação dos princípios fundamentais da contabilidade, sendo aceito pelos órgãos de fiscalização.

O sistema baseado em atividades, também conhecido como ABC (Activity Based Costing), tem a finalidade de não apropriar os custos indiretos pelos rateios tradicionais, mas, sim, pelas atividades envolvidas no processo do negócio. Esse sistema foi criado 
Análise da Eficiência da Gestão de Custos do Processo de Produção de Arroz por pequenos e médios produtores de Limoeiro do Norte - CE

Geanne Benevides Sá Negreiros, Rosângela Venâncio Nunes Rosângela Venâncio Nunes, Charles Washington Costa de Assis, Alexandra Alencar Siebra, Rita de Cássia Fonseca

como instrumento para auxiliar na tomada de decisão, pois, dessa forma, os custos das atividades ficam mais visíveis e identificáveis (Santos, 2011).

O método das unidades de esforço de produção (UEP), conforme descreve Bornia (2010), trabalha apenas com os custos de transformação; já os custos com matéria prima devem ser analisados separadamente, pois não são verificados pelo método. Esse método tem como base a unificação da produção para simplificar o processo de controle de gestão.

\subsection{Estudos Bibliométricos}

A bibliometria tem como objetivo estudar os aspectos quantitativos da produção, da disseminação e do uso da informação registrada. O uso da palavra bibliometria se popularizou a partir de 1969, quando Alan Pritchard sugeriu que ela deveria substituir o termo bibliografia estatística. A bibliometria desenvolve padrões e modelos matemáticos para medir os processos, usando seus resultados para elaborar previsões e apoiar tomadas de decisão (Macias-Chapula, 1998).

Conforme Vanti (2002), o uso das técnicas bibliométricas contribuiu de forma definitiva em épocas de recursos escassos, quando o bibliotecário deveria decidir que títulos e publicações podem ser eliminados de uma biblioteca. Dessa forma, eram obtidos indicadores de uso para definir, por meio de uma lista de publicações periódicas, quais são prioritárias e, dessa maneira, prever a demanda futura.

A bibliometria também era utilizada pelos bibliotecários com a finalidade de calcular quantos profissionais são necessários para atender a um determinado número de leitores. Apesar de ser utilizada para essa finalidade, a bibliometria tem bases mais formais, sofisticadas e estruturadas que produziram resultados relevantes para a filosofia e outras áreas do conhecimento (Braga, 1974).

Araújo (2006) destaca que a bibliometria, inicialmente, era voltada para a medida de livros; posteriormente, foi aos poucos se voltando para estudos dos formatos de produção bibliográfica, como artigos de periódicos e outros tipos de documentos, para depois ocupar-se da produtividade de autores e do estudo de citações. 
Análise da Eficiência da Gestão de Custos do Processo de Produção de Arroz por pequenos e médios produtores de Limoeiro do Norte - CE

Geanne Benevides Sá Negreiros, Rosângela Venâncio Nunes Rosângela Venâncio Nunes, Charles Washington Costa de Assis, Alexandra Alencar Siebra, Rita de Cássia Fonseca

Em relação à bibliometria, Kobashi e Santos (2008, p.109) comentam que se trata de uma metodologia de pesquisa das atividades científicas e correlatas, por meio de análise de dados que apresentem as mesmas particularidades. Como exemplo, podem ser destacadas a identificação da quantidade de trabalhos sobre um determinado assunto, as publicações em uma data precisa, as publicações relativas a um autor ou uma instituição ou difundidas por um periódico científico.

Os estudos bibliométricos, no caso das publicações científicas, têm por finalidade conhecer todos os autores que trabalham em determinado assunto, os periódicos que publicaram sobre esse assunto, os autores considerados referência no assunto, os autores que publicaram em determinado periódico, entre outros, e cruzar todas essas informações com o intuito de aprofundar-se na temática estudada (Kobashi \& Santos, 2008).

Para o melhor entendimento ao que diz respeito à bibliometria, Vanti (2002) descreve algumas possibilidades de aplicação da mesma, como: (a) identificar as tendências e o crescimento do conhecimento em uma área; (b) identificar as revistas do núcleo de uma disciplina; (c) mensurar a cobertura das revistas secundárias; (d) Identificar os usuários de uma disciplina; (e) prever as tendências da publicação; (f) estudar a dispersão e a obsolescência da literatura científica; (g) prever a produtividade de autores individuais, organizações e países; (h) medir o grau e padrões de colaboração entre autores; (i) analisar os processos de citação e cocitação; (j) determinar o desempenho dos sistemas de recuperação da informação; (k) avaliar os aspectos estatísticos da linguagem, das palavras e das frases; (I) avaliar a circulação e o uso de documentos em um centro de documentação; $(\mathrm{m})$ medir o crescimento de determinadas áreas e o surgimento de novos temas.

Santos e Rausch (2009) mencionam, em relação à bibliometria, que esta pode ser considerada como uma ferramenta eficaz na gestão da informação que utiliza inúmeros indicadores de produtividade nos diversos dados abordados. Dessa forma, entre as vantagens que esse método apresenta, pode-se descrever o fato de amenizar 
Análise da Eficiência da Gestão de Custos do Processo de Produção de Arroz por pequenos e
médios produtores de Limoeiro do Norte - CE

Geanne Benevides Sá Negreiros, Rosângela Venâncio Nunes Rosângela Venâncio Nunes, Charles Washington Costa de Assis, Alexandra Alencar Siebra, Rita de Cássia Fonseca

os elementos de julgamento e produzir resultados quantitativos, os quais tendem a ser a soma de pequenos julgamentos e apreciações realizadas por diversas pessoas.

\subsection{Redes Sociais}

As redes sociais também possibilitam a troca de informações com o ambiente que nos cerca. A Análise das Redes Sociais (ARS), também conhecida pela expressão inglesa Social Network Analysis (SNA), é utilizada pelos pesquisadores de diversos campos do conhecimento com a finalidade de compreender o seu impacto sobre a vida social, os quais deram origem a diversas metodologias de análise que têm como base as relações entre os indivíduos, em uma estrutura em forma de redes (Silva, Matheus, Parreiras, \& Parreiras, 2006).

As redes sociais referem-se a pessoas, grupos, instituições, organizações ou outras entidades sociais, conectadas por meio de relacionamentos sociais que envolvam relações de trabalho ou compartilhamento de informações e que são responsáveis por construir ou reconstruir, por meio dessas ligações, uma estrutura social (Machado, Silva, \& Beuren, 2012).

Para Silva et al. (2006), "as redes são sistemas compostos por 'nós' e conexões entre eles, que, nas ciências sociais, são representados por sujeitos sociais (indivíduos, grupos, organizações, etc.) conectados por algum tipo de relação". Tomaél, Alcará e Di Chiara (2005) explicam que, "nas redes sociais, cada indivíduo tem sua função e identidade cultural. Sua relação com outros indivíduos vai formando um todo coeso que representa a rede. De acordo com a temática da organização da rede, é possível a formação de configurações diferenciadas e mutantes".

Tomaél, Alcará e Di Chiara (2005) mencionam que as redes se referem aos agrupamentos e caracterizam-se como fenômenos coletivos, ou seja, representam o relacionamento de grupos, pessoas, organizações ou comunidades, os quais são denominados atores. Dentro do ambiente organizacional, essas redes podem ser usadas como espaços pelos quais ocorre o compartilhamento de informação e do conhecimento. Estas podem surgir por meio de uma conversa informal com um colega 
Análise da Eficiência da Gestão de Custos do Processo de Produção de Arroz por pequenos e
médios produtores de Limoeiro do Norte - CE

Geanne Benevides Sá Negreiros, Rosângela Venâncio Nunes Rosângela Venâncio Nunes, Charles Washington Costa de Assis, Alexandra Alencar Siebra, Rita de Cássia Fonseca

de trabalho, um encontro com amigos, reuniões, congressos, lista de discussões e portais corporativos, com o intuito de alcançar resultados específicos.

As redes sociais ultrapassam 0 ambiente acadêmico ou científico; têm conquistado e ganho espaço em outras esferas. O uso dos recursos gera uma rede em que membros convidam seus amigos, clientes, fornecedores, conhecidos e outros contatos para participar de sua rede, desenvolvendo, dessa forma, uma rede profissional e pessoal. Nesse sentido, emergem ambientes que possibilitam a formação de grupos de interesses que interagem por meio de relacionamentos comuns (Tomaél, Alcará, \& Di Chiara, 2005).

De acordo com Corrêa e Vale (2014), "a análise da evolução das redes de relacionamento de um empreendedor, ao longo de um período, poderia permitir identificar sua trajetória e identificar dimensões importantes do fenômeno de criação e desenvolvimento de seu empreendimento". Dessa forma, a análise das redes sociais ou sociometria pode ser analisada por meio da relação entre um conjunto de atores e seus laços que podem ser fortes ou fracos. Os primeiros demonstram que as informações compartilhadas entre os atores serão as mesmas; por outro lado, os laços fracos atingem contatos indiretos que podem fornecer fontes de informações diferentes e tornam a rede propícia a inovação (Walter, Bach, \& Barbosa, 2012).

Tomaél, Alcará, Di Chiara (2005) comentam que a troca de informações constantes possibilita mudanças essenciais, e essas mudanças correspondem ao conhecimento, pois, quanto mais informações trocamos com o ambiente que nos cerca e com os atores da nossa rede, maior será nossa bagagem de conhecimento e informação, permitindo, dessa forma, a inovação. É nesse sentido que se inserem as

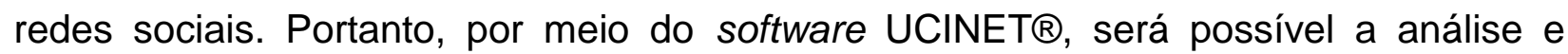
interpretação dos dados coletados.

\subsection{Estudos Anteriores}

$\mathrm{Na}$ literatura foram localizados estudos empíricos que se assemelham ao objetivo desta pesquisa, como a pesquisa realizada por Cruz, Marques, Silva e Cogan 
Análise da Eficiência da Gestão de Custos do Processo de Produção de Arroz por pequenos e
médios produtores de Limoeiro do Norte - CE

Geanne Benevides Sá Negreiros, Rosângela Venâncio Nunes Rosângela Venâncio Nunes, Charles Washington Costa de Assis, Alexandra Alencar Siebra, Rita de Cássia Fonseca

(2010), que objetivou analisar os trabalhos científicos que abordaram a Teoria das Restrições, apresentados no Congresso Brasileiro de Custos, no período de 1994 a 2008, por meio de um estudo Bibliométrico. Os principais resultados desses autores apontaram que, dos 47 artigos analisados, eles apresentavam, em sua maioria, dois autores pertencentes ao gênero masculino, além de pesquisas que se concentravam nas regiões sul e sudeste (92,6\%). Observaram também que a Universidade do Rio de Janeiro foi a instituição mais recorrente, e os autores mais prolíficos foram: Samuel Cogan, Joanília Neide de Sales Cia e Anselmo Rocha Neto. Os enfoques mais abordados buscaram comparar ou integrar as premissas da Teoria das Restrições com outros métodos de gestão de custos, bem como a estratégia de pesquisa mais adotada foi o estudo de caso. Destaca-se que a maioria dos trabalhos é de natureza descritiva ou exclusivamente teórica, bem como as referências utilizadas para a elaboração desses artigos, em sua maioria, são livros (15,7\%).

Nessa mesma linha, Machado, Silva e Beuren (2012) realizaram pesquisa para identificar as características da produção científica de custos, publicada nos periódicos nacionais de contabilidade listados no Qualis/CAPES. Trata-se de pesquisa descritiva com abordagem quantitativa realizada por meio de um estudo bibliométrico e de formação de redes. Nesse trabalho, foram analisados 80 artigos científicos encontrados em 9 periódicos de contabilidade. Os achados revelaram que as temáticas em custos abordaram: métodos de custeio (23 artigos); custos para planejamento e controle (20 artigos); aplicações em custos (19 artigos); abordagem contemporânea (15 artigos); e a temática de ensino e pesquisa ( 5 artigos). Com relação aos métodos de pesquisa, o estudo de caso foi predominante, utilizado em 29 artigos, seguido de revisão bibliográfica em 21 artigos. Com um número reduzido de utilização, consta a survey em 10 artigos. Em relação às redes sociais, essas estão em fase embrionária, prevalecendo as pequenas redes, sem consideração aos centros de distribuição de informações nas pesquisas publicadas.

Moraes Júnior, Araújo e Rezende (2013) realizaram levantamento das pesquisas científicas publicadas no período de 2007 a 2009, na área de "ensino e pesquisa na 
Análise da Eficiência da Gestão de Custos do Processo de Produção de Arroz por pequenos e médios produtores de Limoeiro do Norte - CE

Geanne Benevides Sá Negreiros, Rosângela Venâncio Nunes Rosângela Venâncio Nunes, Charles Washington Costa de Assis, Alexandra Alencar Siebra, Rita de Cássia Fonseca

gestão de custos", veiculadas no Congresso Brasileiro de Custos (CBC). Os achados revelaram que, dentre os 15 artigos científicos analisados, 51\% adotaram abordagem quantitativa, 33\% realizaram análises documentais e $63 \%$ foram pesquisas descritivas.

No estudo de Barboza et al. (2014), o foco é analisar o perfil da produção bibliométrica publicada no âmbito do Congresso Brasileiro de Custos, no período de 1994 a 2012. Foi utilizada a análise quantitativa de dados norteada pelo levantamento bibliográfico. Nessa pesquisa, os autores analisaram 35 artigos que utilizaram a bibliometria para análise da produção científica. Os principais resultados evidenciaram que a pesquisa bibliométrica foi identificada pela primeira vez no ano de 2007, e, nos anos seguintes, não manteve uma regularidade, apresentando-se de forma assimétrica. Prevaleceram as publicações em coautoria e a predominância de referência a livros, sobressaindo a pesquisa descritiva no período estudado. Destacaram que a produção bibliométrica na área contábil, publicada no Congresso Brasileiro de Custos, encontrase em fase de amadurecimento, totalizando um número máximo de duas publicações por autor, como constatado. Ainda observaram a variabilidade das temáticas abordadas dentro do período pesquisado, em que a bibliometria se fez presente em dez áreas temáticas.

Bastos Júnior, Rocha, Cruz, Aires e Munhoz Júnior (2015) investigaram a publicação científica dos pesquisadores dos Programas de Pós-Graduação em Contabilidade da UFSC nos períodos de 2007 a 2012, sob suas perspectivas relacionais e de desempenho, conforme pontuação Qualis/CAPES. Com base na análise do Currículo Lattes dos pesquisados e por meio de métodos sociométricos, bibliométricos, análise de redes e demais abordagens estatísticas, os autores identificaram que o Programa conta com 12 pesquisadores, os quais apresentaram um total de 1.277 publicações científicas, distribuídas entre Anais de Congressos e periódicos. Essas publicações representaram uma pontuação de 2.630 no primeiro triênio, período de 2007 a 2009, e 5.040 no segundo triênio, período de 2010 a 2012. Tais pontuações foram analisadas de forma individual, por pesquisador, com os indicadores de centralidade de redes, possibilitando, dessa forma, realizar uma análise 
Análise da Eficiência da Gestão de Custos do Processo de Produção de Arroz por pequenos e médios produtores de Limoeiro do Norte - CE

Geanne Benevides Sá Negreiros, Rosângela Venâncio Nunes Rosângela Venâncio Nunes, Charles Washington Costa de Assis, Alexandra Alencar Siebra, Rita de Cássia Fonseca

descritiva integrada sobre o desempenho e em pontos dos pesquisadores e suas perspectivas relacionais. Dessa análise, os autores perceberam uma predominante associação direta e persistente entre os indicadores de relacionamento dos pesquisadores e seu desempenho em pontos.

\section{METODOLOGIA DA PESQUISA}

Esta pesquisa se caracteriza como descritiva, uma vez que se buscou analisar as características da produção científica publicadas na revista $A B C u s t o s$, no período de 2006 a 2015, sob a perspectiva das redes sociais e da bibliometria, com abordagem quantitativa dos dados. Esse estudo classificou-se também como uma pesquisa de cunho bibliográfico. Silva (2006) afirma que a pesquisa bibliográfica tem o intuito de explicitar e discutir um tema ou problema a partir de uma base de referências teóricas publicadas em livros, revistas, artigos científicos, entre outros.

A delimitação da população deste estudo restringe-se aos artigos científicos publicados na Revista ABCustos, no período de 2006 a 2015. A amostra é composta de 26 artigos, mapeados a partir da ocorrência da terminologia "gestão de custos", no título, resumo e/ou palavras-chave dos arquivos disponíveis no sítio eletrônico do período analisado.

Após a coleta de dados, realizou-se a análise documental para coletar as informações dispostas nos 26 artigos científicos analisados. Nessa coleta, elaborou-se uma ficha padronizada com os seguintes itens: (a) autores dos artigos; (b) gênero; (c) vínculo institucional dos autores; (d) estado ao qual pertencem as instituições; (e) autoria individual ou múltipla; (f) área de formação dos autores; (g) título do artigo analisado; (h) ano de publicação da revista; (i) temas das pesquisas; (j) método de pesquisa empregado no estudo; (I) classificação das pesquisas em teóricas ou empíricas; $(m)$ métodos estatísticos utilizados nas pesquisas; e $(n)$ referências utilizadas nos artigos. 
Análise da Eficiência da Gestão de Custos do Processo de Produção de Arroz por pequenos e
médios produtores de Limoeiro do Norte - CE

Geanne Benevides Sá Negreiros, Rosângela Venâncio Nunes Rosângela Venâncio Nunes, Charles Washington Costa de Assis, Alexandra Alencar Siebra, Rita de Cássia Fonseca

$\mathrm{Na}$ análise e interpretação dos dados, realizou-se a análise de conteúdo e buscou-se o auxílio da estatística descritiva e nuvem de palavras para a quantificação da produção científica disponível na Revista ABCustos, bem como, para a análise das redes sociais, foi utilizado o software UCINET®.

\section{ANÁLISE DOS RESULTADOS}

Neste tópico, apresentam-se os resultados do estudo a partir do perfil dos autores que publicaram na revista ABCustos, as características dos temas abordados nas pesquisas, as redes sociais dos autores analisados, os métodos empregados nas pesquisas empíricas analisadas e, por fim, as categorias referenciais dos estudos publicados no período de 2006 a 2015.

\subsection{Perfil dos Autores}

Apresentam-se as características que envolvem os 78 autores dos 26 artigos científicos publicados na Revista ABCustos, no período de 2006 a 2015, os quais foram selecionados pela ocorrência da terminologia "Gestão de Custos". Destaca-se que, dentre os 26 estudos empíricos que compõem a amostra final do estudo, apenas dois estudos são de autoria individual, e os outros 24 apresentam autoria múltipla, que compõem uma população de 78 autores responsáveis pelas pesquisas analisadas. Esses achados confirmam os resultados de Barboza et al. (2014) em relação à predominância de estudos empíricos com autoria múltipla quando analisaram as publicações das 19 edições do Congresso Brasileiro de Custos.

Destaca-seque $38,5 \%$ dos autores analisados pertencem ao gênero feminino e $61,5 \%$ ao gênero masculino. Na sequência, a Tabela 1 demonstra o ranking das instituições em que os autores se encontram vinculados. 

Análise da Eficiência da Gestão de Custos do Processo de Produção de Arroz por pequenos e
médios produtores de Limoeiro do Norte - CE

Geanne Benevides Sá Negreiros, Rosângela Venâncio Nunes Rosângela Venâncio Nunes, Charles Washington Costa de Assis, Alexandra Alencar Siebra, Rita de Cássia Fonseca

Tabela 1

Ranking dos autores mais prolíficos e suas respectivas instituições

\begin{tabular}{|c|c|c|}
\hline Ranking & Universidades & Número de Autores \\
\hline 10 & $\begin{array}{l}\text { Universidade Federal do Paraná (UFPR) } \\
\text { Universidade Federal do Rio de Janeiro (UFRJ) }\end{array}$ & 5 \\
\hline $2^{\circ}$ & $\begin{array}{l}\text { Universidade Federal de Uberlândia (UFU) } \\
\text { Universidade Federal de Santa Catarina (UFSC) }\end{array}$ & 4 \\
\hline $3^{0}$ & $\begin{array}{l}\text { Faculdade Dom Alberto (FDA) } \\
\text { Universidade Estadual do Centro Oeste (UNICENTRO) } \\
\text { Universidade Federal do Rio Grande do Sul (UFRGS) }\end{array}$ & 3 \\
\hline $4^{\circ}$ & $\begin{array}{l}\text { Centro Universitário Estácio do Ceará (FIC) } \\
\text { Centro Universitário Franciscano (UNIFRA) } \\
\text { Universidade Regional de Blumenau (FURB) } \\
\text { Serviço Nacional de Aprendizagem Comercial (SENAC) } \\
\text { Universidade do Estado de Santa Catarina (UDESC) } \\
\text { Universidade Federal do Ceará (UFC) } \\
\text { Universidade Federal do Sergipe (UFS) } \\
\text { Universidade Federal da Paraíba (UFPB) } \\
\text { Universidade do Vale do Rio dos Sinos (UNISINOS) }\end{array}$ & 2 \\
\hline
\end{tabular}

Nota. Fonte: Elaborada pelos autores.

Observa-se, na Tabela 1, que, entre as 49 intituições de ensino superior em que os 78 autores estão vinculados atualmente, a Universidade Federal do Paraná (UFPR) e a Universidade Federal do Rio de Janeiro (UFRJ) ocupam a primeira posição com 5 autores respectivamente. Em segundo lugar, com 4 autores, tem-se a Universidade Federal de Uberlândia (UFU) e a Universidade Federal de Santa Catarina (UFSC). Na terceira posição, com 3 autores, aparecem a Faculdade Dom Alberto (FDA), a Universidade Estadual do Centro Oeste (UNICENTRO) e a Universidade Federal do Rio Grande do Sul (UFRGS).

Percebe-se que $62 \%$ dos autores que publicaram sobre o assunto possuem 0 título de doutor e $24 \%$ de mestre. Em quantidades menos expressivas, tem-se $8 \%$ com pós-graduação e 6\% dos autores com graduação. Destaca-se também, no referente à área de formação dos autores, que o doutorado em Administração é o que mais se 
Análise da Eficiência da Gestão de Custos do Processo de Produção de Arroz por pequenos e médios produtores de Limoeiro do Norte - CE

Geanne Benevides Sá Negreiros, Rosângela Venâncio Nunes Rosângela Venâncio Nunes, Charles Washington Costa de Assis, Alexandra Alencar Siebra, Rita de Cássia Fonseca

destaca, com um total de 29,17\%. Na formação de mestrado, $84 \%$ dos autores apresentam formação na área contábil. No nível de especialização, aparecem: Auditoria, MBA em Gerência Contábil, Logística e Gestão Econômica e Financeira, Controladoria e Finanças. Ainda, no nível de graduação, aparece o curso de Bacharel em Ciências Contábeis.

Quanto à distribuição dos autores por região geográfica, observa-se a predominância das regiões sul (56\%), sudeste $(23 \%)$ e nordeste $(21 \%)$ entre os autores analisados. Na região sul, o estado de Santa Catarina (24\%) apresenta o maior número de autores que publicaram no periódico analisado, seguido do Rio Grande do Sul (21\%) e Paraná (12\%). Em seguida, a região sudeste possui $23 \%$ do total dos autores, englobando os estados do Espírito Santo, Minas Gerais, São Paulo e Rio de Janeiro. Por fim, a região nordeste tem $21 \%$ dos autores que publicam na ABCustos, representada pelos estados da Bahia, Ceará, Paraíba, Pernambuco e Sergipe.

\subsection{Temas Abordados pelos Estudos}

Inicia-se a apresentação dos resultados com os dados acerca da evolução das publicações sobre o tema no período analisado, conforme explicita a Tabela 2. 
Análise da Eficiência da Gestão de Custos do Processo de Produção de Arroz por pequenos e médios produtores de Limoeiro do Norte - CE

Geanne Benevides Sá Negreiros, Rosângela Venâncio Nunes Rosângela Venâncio Nunes, Charles Washington Costa de Assis, Alexandra Alencar Siebra, Rita de Cássia Fonseca

Tabela 2

Quantidade de artigos por ano

\begin{tabular}{c|c|c}
\hline Ano & Quantidade de artigos & Percentual \\
\hline 2006 & 0 & $0 \%$ \\
\hline 2007 & 2 & $8 \%$ \\
\hline 2008 & 1 & $4 \%$ \\
\hline 2009 & 3 & $12 \%$ \\
\hline 2010 & 7 & $27 \%$ \\
\hline 2011 & 2 & $8 \%$ \\
\hline 2012 & 4 & $15 \%$ \\
\hline 2013 & 2 & $8 \%$ \\
\hline 2014 & 3 & $12 \%$ \\
\hline 2015 & 2 & $8 \%$ \\
\hline Total & $\mathbf{2 6}$ & $\mathbf{1 0 0} \%$ \\
\hline
\end{tabular}

Nota. Fonte: Elaborada pelos autores.

Observa-se, na Tabela 2, que o ápice de publicações na Revista ABCustos no período de 2006 a 2015 ocorreu no ano de 2010, com sete estudos que abordaram a "Gestão de Custos". Em seguida, verifica-se que as publicações têm-se mantido constantes ao longo dos anos, porém em menor número, pois em 2011, 2013 e 2015 são mapeadas duas publicações; 2012 aparece com quatro publicações e 2014 com três artigos científicos publicados.

$\mathrm{Na}$ sequência, na Figura 1 tem-se a representação gráfica da ocorrência dos enfoques temáticos dos 26 estudos empíricos que abordam a gestão de custos publicados na Revista ABCustos. 
Análise da Eficiência da Gestão de Custos do Processo de Produção de Arroz por pequenos e médios produtores de Limoeiro do Norte - CE

Geanne Benevides Sá Negreiros, Rosângela Venâncio Nunes Rosângela Venâncio Nunes, Charles Washington Costa de Assis, Alexandra Alencar Siebra, Rita de Cássia Fonseca

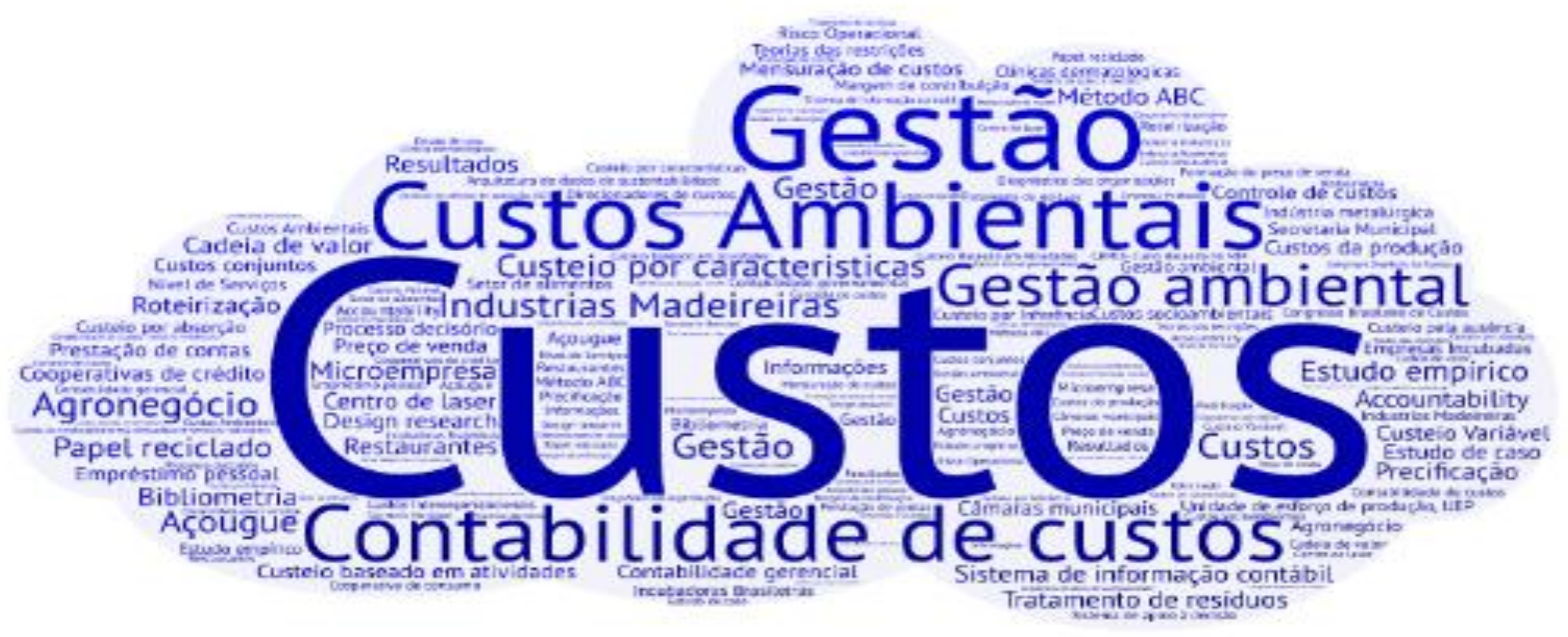

Figura 1. Representação gráfica dos temas abordados nas pesquisas analisadas Fonte: Elaborada pelos autores.

A partir dos 26 estudos empíricos analisados, a terminologia mais utilizada foi "custos", seguida pela palavra "gestão", "custos ambientais" e "contabilidade de custos", conforme demonstra análise realizada por meio da nuvem de palavras. Destaca-se que a análise considerou 83 palavras-chave apresentadas nos artigos científicos analisados, e a nuvem de palavra considera o número de ocorrência dessas terminologias para gerar a representação gráfica.

Esses resultados confirmam os achados de Barboza et al. (2014), uma vez que as terminologias "Contabilidade", "Gestão de Custos", "Custos Ambientais" foram as mais recorrentes entre as palavras-chave dos estudos publicados nas 19 edições do Congresso Brasileiro de Custos.

\subsection{Redes Sociais dos Autores}

$\mathrm{Na}$ Figura 2, evidenciam-se as redes sociais formadas pelos 78 autores mapeados a partir da produção científica na Revista ABCustos, no período de 2006 a 2015, que realizam estudos voltados à gestão de custos. 
Análise da Eficiência da Gestão de Custos do Processo de Produção de Arroz por pequenos e médios produtores de Limoeiro do Norte - CE

Geanne Benevides Sá Negreiros, Rosângela Venâncio Nunes Rosângela Venâncio Nunes, Charles Washington Costa de Assis, Alexandra Alencar Siebra, Rita de Cássia Fonseca

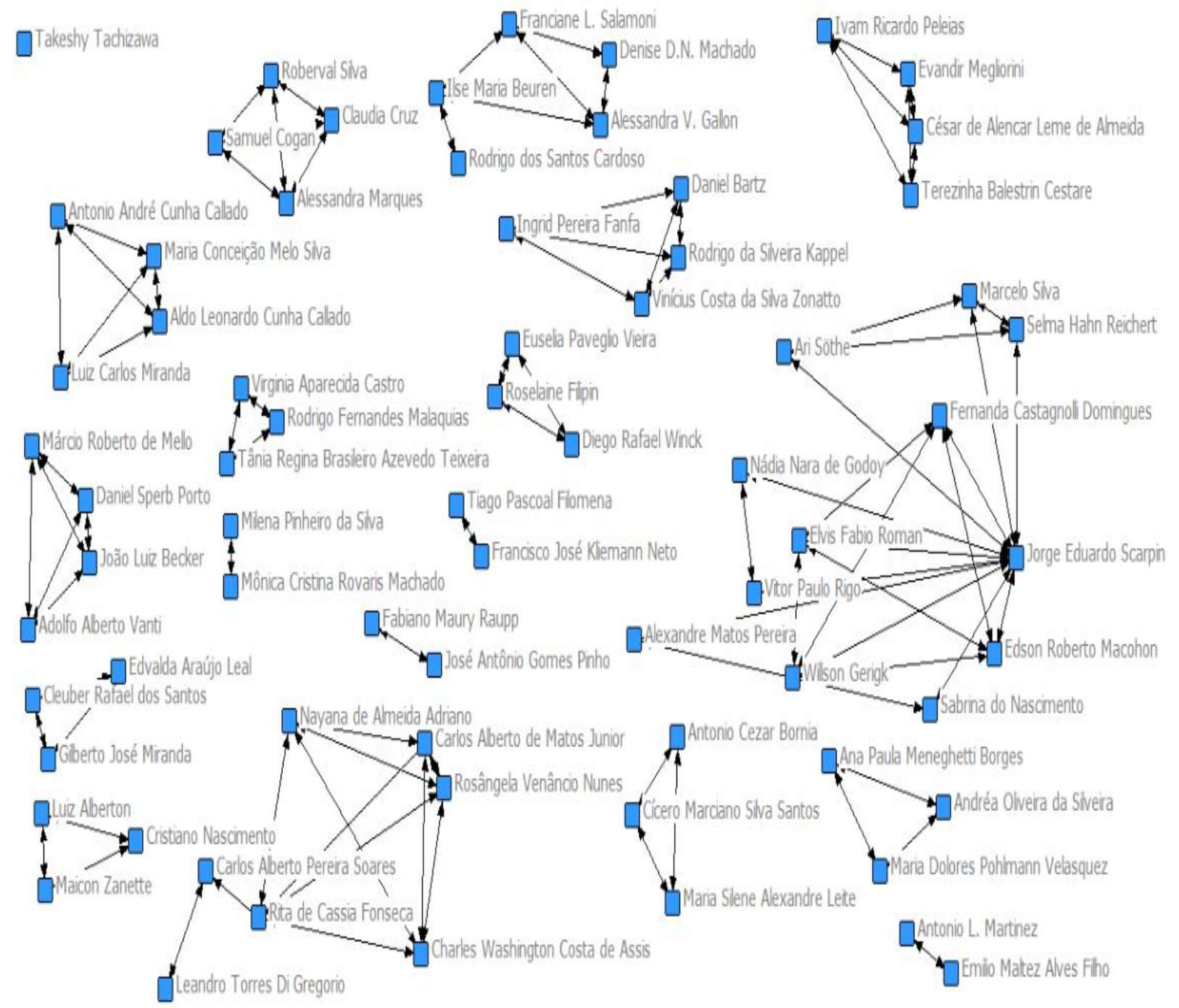

Figura 2. Redes sociais dos autores que realizam estudos voltados à gestão de custos publicados na Revista ABCustos, no período de 2006 a 2015 Fonte: Elaborada pelos autores.

Percebe-se, na Figura 2, que a rede de autores mapeados nos 26 estudos analisados, que a posição central na rede social é ocupada pelo professor doutor Jorge Eduardo Scarpin, com quatro estudos de autoria múltipla publicados no periódico. Destacam-se também os autores Alessandra Vasconcelos Gallon, Fabiano Maury 
Análise da Eficiência da Gestão de Custos do Processo de Produção de Arroz por pequenos e
médios produtores de Limoeiro do Norte - CE

Geanne Benevides Sá Negreiros, Rosângela Venâncio Nunes Rosângela Venâncio Nunes, Charles Washington Costa de Assis, Alexandra Alencar Siebra, Rita de Cássia Fonseca

Raupp, Franciane Luiza Salamoni e Ilse Maria Beuren, com 2 estudos publicados no período.

Outro aspecto relevante, apontado na Figura 2, corresponde à presença de uma lacuna estrutural e laços fracos, pois os autores não realizam o compartilhamento de informações com o grupo, tendo em vista o elevado número de autores que deixaram de publicar seus estudos científicos com os outros autores que publicaram na Revista ABCustos, embora os autores, em sua maioria, residem nas regiões sul e sudeste do país. Destaca-se ainda que o autor Takeshy Tachizawa não interagiu com nenhum outro autor, por essa questão aparecer isolada na rede social. Para Burt (1992), a presença de lacunas estruturais se apresenta como uma vantagem competitiva para os atores que atuaram na rede social e realizaram a conexão entre as diferentes redes, já que os atores não conectados não efetuaram compartilhamento de informações com os demais atores.

Os achados deste estudo corroboram os resultados da pesquisa realizada por Machado, Silva e Beuren (2012), que analisaram a produção científica de custos em periódicos de contabilidade. Os autores evidenciaram que existe um número de pesquisadores que ainda carecem de integração, pois apresentaram baixa representação na forma de centralidade da rede social. Estes desenvolveram pesquisas isoladas sobre a temática de custos. Além disso, tem-se um vasto número de redes com três pesquisadores em cinco grupos e quatorze redes com dois pesquisadores que não configuram o entrelaçamento da rede.

\subsection{Métodos Empregados nas Pesquisas Analisadas}

Em relação ao método de pesquisa, percebe-se, dentre a natureza dos estudos analisados, que $77 \%$ são de natureza empírica e $26 \%$ são de natureza teórica. Quanto à abordagem do problema, tem-se a predominância da abordagem qualitativa (54\%), seguida da quantitativa (35\%) e qualitativa-quantitativa (12\%). Ainda, no que se refere à natureza do objetivo, a pesquisa descritiva foi predominante com $54 \%$ dos estudos; já 

Análise da Eficiência da Gestão de Custos do Processo de Produção de Arroz por pequenos e
médios produtores de Limoeiro do Norte - CE

Geanne Benevides Sá Negreiros, Rosângela Venâncio Nunes Rosângela Venâncio Nunes, Charles Washington Costa de Assis, Alexandra Alencar Siebra, Rita de Cássia Fonseca

as pesquisas exploratórias corresponderam a 35\% dos artigos analisados, e as exploratório-descritivas, a 12\%.

Os resultados coadunam com os achados de Barboza et al. (2014), cujas pesquisas descritivas representaram $61 \%$ dos estudos analisados; as pesquisas exploratórias, 12\%; e as descritivo-exploratórias, 9\%. E os achados de Moraes Júnior, Araújo e Rezende (2013), em que os estudos descritivos representaram 73\% dos artigos científicos investigados, apresentaram a predominância de artigos com abordagem do problema quantitativa.

Em seguida, a Tabela 3 explicita as técnicas de coletas de dados utilizadas pelos 26 artigos pesquisados.

Tabela 3

Técnica de coleta de dados dos estudos empíricos analisados

\begin{tabular}{l|c|c}
\hline \multicolumn{1}{c|}{ Técnica de coleta de dados } & Quantidade & Percentual \\
\hline Análise documental & 11 & $42 \%$ \\
\hline Análise documental e observação & 1 & $4 \%$ \\
\hline Base de dados Economática & 1 & $4 \%$ \\
\hline Entrevista & 2 & $8 \%$ \\
\hline Entrevista e análise documental & 2 & $8 \%$ \\
\hline Entrevista semiestruturada e observação & 1 & $4 \%$ \\
\hline Entrevista semiestruturada e questionário & 1 & $4 \%$ \\
\hline Observação, entrevista informal e análise documental & 1 & $4 \%$ \\
\hline Questionário & 4 & $15 \%$ \\
\hline Questionário e análise documental & 1 & $4 \%$ \\
\hline Visitas in loco e análise documental & 1 & $4 \%$ \\
\hline Total & $\mathbf{2 6}$ & $\mathbf{1 0 0 \%}$ \\
\hline
\end{tabular}

Nota. Fonte: Elaboradora pelos autores.

Verifica-se, na Tabela 3, a predominância da análise documental (42\%) como técnica de coleta de dados para elaboração dos estudos empíricos analisados. $\mathrm{Na}$ segunda posição, aparecem os questionários com 15\% e as entrevistas (8\%), bem como as entrevistas que, quando são somadas a outras técnicas, representam $24 \%$. 
Análise da Eficiência da Gestão de Custos do Processo de Produção de Arroz por pequenos e médios produtores de Limoeiro do Norte - CE

Geanne Benevides Sá Negreiros, Rosângela Venâncio Nunes Rosângela Venâncio Nunes, Charles Washington Costa de Assis, Alexandra Alencar Siebra, Rita de Cássia Fonseca

Na sequência, a Tabela 4 apresenta a natureza do método mapeado nos 26 artigos analisados.

Tabela 4

Natureza do método de pesquisa empírica adotado nos estudos pesquisados

\begin{tabular}{l|c|c}
\hline \multicolumn{1}{c|}{ Tipo de método } & Quantidade & Percentual \\
\hline Conceitual & 4 & $15 \%$ \\
\hline Design Research & 1 & $4 \%$ \\
\hline Estudo de caso & 11 & $42 \%$ \\
\hline Estudo de múltiplos casos & 2 & $8 \%$ \\
\hline GroundedTheory & 1 & $4 \%$ \\
\hline Levantamento ou Survey & 4 & $15 \%$ \\
\hline Pesquisa Ação & 1 & $4 \%$ \\
\hline Pesquisa documental & 2 & $\mathbf{8 \%}$ \\
\hline Total & $\mathbf{2 6}$ & $\mathbf{1 0 0 \%}$ \\
\hline
\end{tabular}

Nota. Fonte: Elaborada pelos autores.

Observa-se que o estudo de caso (42\%) é mais recorrente entre os artigos analisados. Em seguida, aparecem os estudos teóricos de natureza conceitual e as pesquisas de levantamento ou survey presentes em 15\% dos artigos analisados. Esses achados corroboram os de Moraes Júnior, Araújo e Rezende (2013), que também evidenciaram a predominância das pesquisas de levantamento.

Quanto ao local de realização dos estudos, verifica-se uma diversidade de ambientes que compreendem as pesquisas analisadas que vão desde restaurantes, indústrias, cooperativas de crédito, cooperativas de carne, agroindústria, concessionária de energia elétrica, secretaria municipal de agricultura e meio ambiente, secretaria municipal de educação, incubadoras, empresas listadas na BM\&FBOVESPA, empresa agrícola, empresa de serviços rodoviários, clinicas dermatológicas, câmaras municipais e análise de artigos científicos. A Tabela 5 explicita os tipos de análise de dados aplicada na amostra de estudos. 
Análise da Eficiência da Gestão de Custos do Processo de Produção de Arroz por pequenos e médios produtores de Limoeiro do Norte - CE

Geanne Benevides Sá Negreiros, Rosângela Venâncio Nunes Rosângela Venâncio Nunes, Charles Washington Costa de Assis, Alexandra Alencar Siebra, Rita de Cássia Fonseca

Tabela 5

Técnicas de análise dos dados adotados nos estudos pesquisados

\begin{tabular}{l|c|c}
\hline \multicolumn{1}{c|}{ Técnica de análise dos dados } & Quantidade & Percentual \\
\hline Análise de conteúdo & 19 & $\mathbf{7 3 \%}$ \\
\hline Análise multicritério & 1 & $4 \%$ \\
\hline Regressão estatística & 1 & $4 \%$ \\
\hline Análise de agrupamento, escalonamento multidimensional e & 1 & $4 \%$ \\
\hline análise fatorial & 1 & $4 \%$ \\
\hline Simulação de cálculos probabilísticos e matemáticos & 1 & $4 \%$ \\
\hline Entrevista semiestruturada e documentos primários & 1 & $4 \%$ \\
\hline Medições in loco, entrevistas semiestruturadas e & 1 & $4 \%$ \\
\hline documentos & $\mathbf{2 6}$ & $\mathbf{1 0 0 \%}$ \\
\hline Entrevista não-estruturada e análise de conteúdos & & \\
\hline Total & & \\
\hline
\end{tabular}

Nota. Fonte: Elaborada pelos autores.

Observa-se, na Tabela 5, que 73\% dos artigos adotam a análise de conteúdo. As demais técnicas aparecem em quantidades menos relevantes, com 4\% cada. Dentre elas, se encontram: análise multicritério, regressão estatística, análise de agrupamento, escalonamento multidimensional e análise fatorial, simulação de cálculos probabilísticos e matemáticos, entrevista semiestruturada e documentos primários, medições in loco e análise de conteúdo.

\subsection{Categorias Referenciais}

A análise das referências contempladas nos 26 estudos empíricos analisados que abordam a gestão de custos envolveu 545 referências, conforme demonstra a Tabela 6. 
Análise da Eficiência da Gestão de Custos do Processo de Produção de Arroz por pequenos e médios produtores de Limoeiro do Norte - CE

Geanne Benevides Sá Negreiros, Rosângela Venâncio Nunes Rosângela Venâncio Nunes, Charles Washington Costa de Assis, Alexandra Alencar Siebra, Rita de Cássia Fonseca

Tabela 6

Categorias referenciais dos artigos científicos analisados

\begin{tabular}{l|c|c}
\hline Categorias referenciais & Quantidade & Percentual (\%) \\
\hline Artigos publicados em anais de congressos & 56 & $10 \%$ \\
\hline Artigo publicados em periódicos científicos & 27 & $5 \%$ \\
\hline Dissertação/Tese & 32 & $6 \%$ \\
\hline Livros & 291 & $53 \%$ \\
\hline Revista/Jornal & 64 & $12 \%$ \\
\hline Sites & 75 & $14 \%$ \\
\hline Total & $\mathbf{5 4 5}$ & $\mathbf{1 0 0 \%}$ \\
\hline
\end{tabular}

Nota. Fonte: Elaborada pelos autores.

Percebe-se que, dentre as 545 referências mapeadas nos 26 estudos empíricos analisados, tem-se a predominância dos livros, com $53 \%$ das fontes utilizadas para a construção dos trabalhos pesquisados. Em seguida, aparecem os sites (14\%), as revistas e/ou jornais e os artigos científicos publicados em anais de congresso com $14 \%, 12 \%$ e 10\% respectivamente. Observa-se que as dissertações e/ou teses e artigos científicos publicados em periódicos científicos foram utilizados com menor frequência como fonte de consulta para a construção dos estudos analisados.

Os resultados acima coadunam com os achados de Cruz et al. (2010) que analisaram a produção científica voltada à Teoria das Restrições (TOC) no Congresso Brasileiro de Custos, no período de 1994 a 2008, em que a categoria livros foi predominante entre as referências utilizadas. Para os autores, esses resultados apontaram um posicionamento conservador e convencional por parte dos autores na construção de seus referenciais teóricos. Os autores também fizeram pouca consulta a periódicos e anais de congressos de natureza científica. E, ainda, confirmaram os achados de Barboza et al. (2014), que também revelaram o predomíniodos livros como fontes de consulta para elaboração dos artigos publicados nas 19 edições do Congresso Brasileiro de Custos. 
Análise da Eficiência da Gestão de Custos do Processo de Produção de Arroz por pequenos e
médios produtores de Limoeiro do Norte - CE

Geanne Benevides Sá Negreiros, Rosângela Venâncio Nunes Rosângela Venâncio Nunes, Charles Washington Costa de Assis, Alexandra Alencar Siebra, Rita de Cássia Fonseca

Quanto ao título das obras utilizadas para construção da plataforma teórica dos estudos analisados, a Tabela 7 apresenta um ranking do título das obras mais utilizadas.

Tabela 7

Obras mais utilizadas na construção da plataforma teórica dos estudos pesquisados

\begin{tabular}{c|l|l}
\hline Ranking & \multicolumn{1}{|c|}{ Títulos das Obras } & \multicolumn{1}{c}{ Autor (es) } \\
\hline \multirow{2}{*}{0} & Contabilidade de Custos & Eliseu Martins \\
\hline \multirow{3}{*}{20} & Gestão de Custos, Contabilidade e Controle & $\begin{array}{l}\text { Maryane M. Mowen } \\
\text { Don R. Hansen }\end{array}$ \\
\cline { 2 - 3 } & $\begin{array}{l}\text { Gestão de Custos e Formação de Preço: com aplicação da } \\
\text { calculadora HP 12C e Excel. }\end{array}$ & Adriano Leal Bruni \\
\cline { 2 - 3 } & ABC- Custeio Baseado em Atividades & MasayukiNakagawa \\
\cline { 2 - 3 } & Análise Gerencial de Custos em Empresas Modernas & Antônio Cesar Bornia \\
\hline \multirow{2}{*}{$\cong$} & $\begin{array}{l}\text { Contabilidade de Custos: criando valor para a } \\
\text { administração }\end{array}$ & Michael Maher \\
\hline
\end{tabular}

Nota. Fonte: Elaborada pelos autores.

$\mathrm{Na}$ Tabela 7, verifica-se que a obra mais utilizada entre as referências consultadas foi "Contabilidade de Custos", de autoria de Eliseu Martins. Na segunda posição, destacam-se as obras "Gestão de Custos, Contabilidade e controle", "Gestão de Custos e Formação de Preço: com Aplicação da Calculadora HP 12C e Excel", "ABC - Custeio Baseado em Atividades" e "Análise Gerencial de Custos em Empresas Modernas", com autoria de Maryane M. Mowen e Don R. Hansen, Adriano Leal Bruni, Masayuki Nakagawa e Antônio Cesar Bornia, respectivamente. Em terceiro lugar, aparece a obra "Contabilidade de Custos: criando valor para a administração", com autoria de Michael Maher.

\section{CONSIDERAÇÕES FINAIS}

O estudo analisou as características da produção científica voltada à gestão de custos publicada na ABCustos, no período de 2006 a 2015, sob a perspectiva das 
Análise da Eficiência da Gestão de Custos do Processo de Produção de Arroz por pequenos e médios produtores de Limoeiro do Norte - CE

Geanne Benevides Sá Negreiros, Rosângela Venâncio Nunes Rosângela Venâncio Nunes, Charles Washington Costa de Assis, Alexandra Alencar Siebra, Rita de Cássia Fonseca

redes sociais e da bibliometria. A pesquisa se caracteriza como descritiva e de cunho bibliográfico, com análise quantitativa dos dados. A amostra compreende 26 artigos selecionados por meio da ocorrência da terminologia "gestão de custos".

Entre os 26 estudos empíricos analisados, 24 apresentam autoria múltipla, e $61,5 \%$ dos autores pertencem ao gênero masculino. Dentre as instituições de ensino superior nas quais os autores apresentam vínculo institucional, a Universidade Federal do Paraná e a Universidade Federal do Rio de Janeiro são as mais recorrentes. Cabe mencionar que a maioria dos autores analisados reside na região Sul $(56 \%)$, seguindose os das regiões sudeste e nordeste. A formação dos autores corresponde à titulação de doutorado (62\%), sendo a formação em Administração predominante $(29,17 \%)$.

No que se refere ao método de pesquisa utilizado, tem-se a predominância dos estudos empíricos (77\%), com abordagem do problema qualitativa (54\%) e natureza do objetivo descritiva (54\%). Esses resultados corroboram parcialmente a pesquisa de Moraes Júnior, Araújo e Rezende (2013), em que os estudos descritivos são preponderantes, porém com abordagem do problema qualitativa.

Em relação às redes sociais de autoria, tem-se a posição central da rede ocupada pelo professor doutor Jorge Eduardo Scarpin, que está vinculado à Universidade Federal do Paraná. Entretanto, a rede social apresenta uma lacuna estrutural com laços de autoria considerados fracos. Os resultados corroboram os achados de Machado, Silva e Beuren (2012) quando apontam a necessidade de interação entre os autores, a baixa representação na forma de centralidade da rede e o vasto número de redes com pequeno número de atores. Esta situação não configura o entrelaçamento da rede social na área de custos.

Quanto às categorias referenciais, os livros representam $53 \%$ das fontes consultadas para a construção do referencial teórico dos estudos analisados, e a obra mais citada foi "Contabilidade de Custos", de autoria de Eliseu Martins. Verifica-se também a pequena utilização de Dissertações e/ou Teses e artigos científicos publicados em periódicos e anais de congressos científicos. Neste sentido, 78 dos autores analisados apresentam um posicionamento mais conservador e convencional 
Análise da Eficiência da Gestão de Custos do Processo de Produção de Arroz por pequenos e
médios produtores de Limoeiro do Norte - CE

Geanne Benevides Sá Negreiros, Rosângela Venâncio Nunes Rosângela Venâncio Nunes, Charles Washington Costa de Assis, Alexandra Alencar Siebra, Rita de Cássia Fonseca

para a construção de seus referenciais teóricos, além da pouca consulta a periódicos e anais de congressos científicos que coadunam com os achados de Cruz et al. (2010).

Considera-se, a partir da análise dos 26 estudos empíricos analisados que abordam a gestão de custos, no período de 2006 a 2015, que eles demonstram a pouca profundidade dos estudos que tratam da gestão de custos e evidenciam uma lacuna de pesquisa na área contábil relacionada à necessidade de pesquisas empíricas voltadas à gestão de custos no contexto organizacional que subsidiem o processo decisório. Nesse sentido, espera-se que esta pesquisa possa motivar os pesquisadores brasileiros a explorarem as diversas metodologias de pesquisa que podem ser implementadas para a investigação da gestão estratégica de custos.Sugere-se, para futuras pesquisas, a realização de estudos voltados aos temas abordados dentro da Contabilidade de Custos, veiculados nos congressos e periódicos especializados da área.

\section{REFERÊNCIAS}

ABCustos. Foco e escopo. Recuperado de: https://www.abcustos.emnuvens.com.br/ abcustos/about/editorialPolicies\#focusAndScope

Araújo, C. A. (2006). Bibliometria: evolução histórica e questões atuais. Em Questão, 12(1),11-32.

Barboza, M. M. B. M., Souza, W. D., Nascimento, J. C. H. B., Bernardes, J. R., \& Castro, M. B. M. B. (2014). O perfil da pesquisa bibliométrica publicada nas 19 edições do Congresso Brasileiro de Custos. Anais do Congresso Brasileiro de Custos, Natal, Brasil, 21.

Bastos Júnior, E.; Rocha, D. T.; Cruz, J. A. W.; Aires, O. T. A. M. \& Munhoz Junior, J. P. (2015). Relacionamento e desempenho: estudo bibliométrico e sociométrico da produção científica do programa de pós-graduação em contabilidade da UFSC. Pensar Contábil, 17(63), 41-51.

Bornia, A. C. (2010). Análise gerencial de custos: aplicação em empresas modernas. São Paulo: Atlas.

Braga, G. M. (1974). Informação, ciência, política científica: o pensamento de Derek de SollaPrice. Ciência da Informação, 2,155-177. 

Análise da Eficiência da Gestão de Custos do Processo de Produção de Arroz por pequenos e
médios produtores de Limoeiro do Norte - CE

Geanne Benevides Sá Negreiros, Rosângela Venâncio Nunes Rosângela Venâncio Nunes, Charles Washington Costa de Assis, Alexandra Alencar Siebra, Rita de Cássia Fonseca

Burt, R. S. (1992). Structural holes: the social structure of competition. Cambridge: Harvard University Press.

Corrêa, V. S., \& Vale, G. M. V. (2014). Redes sociais, perfil empreendedor e trajetórias. Revista de Administração Contemporânea, 49(1), 77-88.

Cruz, C.; Marques, A.; Silva, R., \& Cogan, S. (2010). Teoria das Restrições: um estudo Bibliométrico da produção científica apresentada no congresso brasileiro de custos (1994-2008). ABCustos, 5(1).

Kobashi, N. Y., \& Santos, R. N. M. (2008). Arqueologia do trabalho imaterial: uma aplicação bibliométrica à análise de dissertações e teses. Enc. Bibli: Revista Eletrônica, Biblioteconomia e Ciência da Informação, n. esp., $1^{\circ}$ sem.

Leone, G. S. G. (2012). Custos: planejamento, implantação e controle. São Paulo: Atlas.

Machado, D. G., Silva, T. P., \& Beuren, I. M. (2012). Produção científica de custos: análise das publicações em periódicos nacionais de contabilidade sob a perspectiva das redes sociais e da bibliometria. Contabilidade, Gestão e Governança. 15(3),316.

Macias-Chapula, C. A. (1998). O papel da infometria e da cienciometria e sua perspectiva nacional e internacional. Ciência da Informação, 27(2),134-140.

Martins, E., \& Rocha, W. (2010). Métodos de custeio comparados:custos e margens analisados sob diferentes perspectivas. São Paulo: Atlas.

Moraes Júnior, V. F., Araújo, A. O. \& Rezende, I. C. C. (2013). Estudo bibliométrico da área ensino e pesquisa em gestão de custos: triênio2007-2009 do Congresso Brasileiro de Custos. Revista de Administração, Contabilidade e Sustentabilidade, 3(2), 20-38.

Ritta, C. O. Citadin, A., \& Pereira, B. S. (2015). Análise da Produção Científica sobre Gestão Estratégica de Custos no Congresso Brasileiro de Custos. Contabilidade e Controladoria do século XVI. Anais do Congresso USP de Iniciação Científica, São Paulo, 12.

Santos, A. F. \& Rausch, R. B. (2009). Perícia Contábil na Revista Brasileira de Contabilidade: uma análise bibliométrica do período de 1992 a 2008. Anais do Congresso Brasileiro de Custos, Fortaleza, 16. 
Análise da Eficiência da Gestão de Custos do Processo de Produção de Arroz por pequenos e
médios produtores de Limoeiro do Norte - CE Geanne Benevides Sá Negreiros, Rosângela Venâncio Nunes Rosângela Venâncio Nunes, Charles Washington Costa de Assis, Alexandra Alencar Siebra, Rita de Cássia Fonseca

Santos, J. J. (2011). Contabilidade de custos e análise de custos.São Paulo: Atlas.

Schier, C. U. C. (2004). Gestão prática de custos.Curitiba: Juruá.

Silva, A. B. O, Matheus, R. F.; Parreiras, F. S., \& Parreiras, T. A. S. (2006). Análise de redes sociais como metodologia de apoio para a discussão da interdisciplinaridade na ciência da informação. Ciência da Informação, 35(1), 72-93.

Silva, A. C. R. (2006). Metodologia da pesquisa aplicada à contabilidade:orientações de estudos, projetos, artigos, relatórios, monografias, dissertações, teses. São Paulo: Atlas.

Silva, R. N. S., \& Lins, L. S. (2010). Gestão de custos: contabilidade, controle e análise. São Paulo: Atlas.

Tomáel, M. I., Alcará, A. R., \& Di Chiara, I. G. (2005). Das redes sociais à inovação. Ciência da Informação, 34 (2), 93-104.

Vanti, N. A. P. (2002). Da bibliometria à webometria: uma exploração conceitual dos mecanismos utilizados para medir o registro da informação e a difusão do conhecimento. Ciência da Informação, 31(2),152-162.

Walter, S. A. Bach, T. M., Barbosa, F. (2012). Estrutura das redes sociais e da bibliometria: uma análise longitudinal da abordagem de estratégia como prática. Anais do Encontro da Associação Nacional dos Programas de Pós-Graduação e Pesquisa em Administração, Rio de Janeiro, 36.

Wernke, R. (2005). Análise de custos e preços de venda: ênfase em aplicações e casos nacionais. São Paulo: Saraiva.

Data de Submissão: 08/12/2016

Data de Aceite: 27/10/2017 